\title{
Targeted therapy of triple negative MDA-MB-468 breast cancer with curcumin delivered by epidermal growth factor-conjugated phospholipid nanoparticles
}

\author{
KYUNG-HO JUNG ${ }^{1,2}$, JIN HEE LEE ${ }^{1,2}$, JIN WON PARK ${ }^{1,2}$, DA HAE KIM ${ }^{1}$, \\ SEUNG-HWAN MOON ${ }^{1}$, YOUNG SEOK CHO ${ }^{1}$ and KYUNG-HAN LEE ${ }^{1,2}$ \\ ${ }^{1}$ Department of Nuclear Medicine, Samsung Medical Center; ${ }^{2}$ Samsung Advanced Institute for Health and \\ Sciences and Technology, Sungkyunkwan University School of Medicine, Seoul 06351, Republic of Korea
}

Received October 10, 2017; Accepted April 5, 2018

DOI: $10.3892 / \mathrm{ol} .2018 .8471$

\begin{abstract}
Triple-negative breast cancer (TNBC) is associated with poor survival as chemotherapy is currently limited to conventional cytotoxic agents. Curcumin has promising anticancer actions against TNBC, but its application is hindered by poor bioavailability and rapid degradation in vivo. In the present study, curcumin-loaded phospholipid nanoparticles (Cur-NPs) conjugated with epidermal growth factor (EGF) were prepared for specific targeting of EGF receptors overexpressed in TNBC. NP formulation was performed by reacting EGF peptide with N-hydroxysuccinimide-Polyethylene Glycol1,2-Distearoyl-sn-Glycero-3-Phosphoethanolamine (NHS$\mathrm{PEG}_{10000}$-DSPE), followed by efficient curcumin loading through lipid film hydration. EGF conjugation did not significantly affect NP size, zeta potential or morphology. Specific targeting was confirmed by EGF receptor activation and blocking of ${ }^{125} \mathrm{I}$-labeled NP binding by excess EGF. EGF-Cur-NP dose-dependently suppressed MDA-MB-468 TNBC cell survival (IC50, $620 \mathrm{nM}$ ), and completely abolished their capacity to form colonies. The cytotoxic effects were more potent compared with those of free curcumin or Cur-NP. In mice bearing MDA-MB-468 tumors, injections of $10 \mathrm{mg} / \mathrm{kg}$ EGF-Cur-NP caused a 59.1\% retardation of tumor growth at 3 weeks compared with empty NP, whereas the antitumor effect of Cur-NP was weak. These results indicate
\end{abstract}

Correspondence to: Professor Kyung-Han Lee, Department of Nuclear Medicine, Samsung Medical Center, 50 Ilwondong Road, Gangnamgu, Seoul 06351, Republic of Korea

E-mail:khnm.lee@samsung.com

Abbreviations: TNBC, triple-negative breast cancer; ER, estrogen receptor; $\mathrm{PR}$, progesterone receptor; HER2, human epidermal growth factor receptor 2; NPs, nanoparticles; EGF, epidermal growth factor; NHS-PEG-DSPE, N-hydroxysuccinimide-Polyethylene Glycol-1,2-Distearoyl-sn-Glycero-3-Phosphoethanolamine

Key words: curcumin, triple negative breast cancer, epidermal growth factor, phospholipid nanoparticle that EGF-conjugated NHS-PEG ${ }_{10000}$-DSPE phospholipid NPs loaded with curcumin may be useful for treating TNBCs that overexpress the EGF receptor.

\section{Introduction}

Triple-negative breast cancers (TNBCs) that lack expression of estrogen receptor (ER), progesterone receptor (PR) and human epidermal growth factor receptor 2 (HER2) account for $15-20 \%$ of all breast cancers (1). Clinically, TNBCs are associated with greater aggressiveness and early metastasis that result in worse patient survival compared to other breast cancer types (2). Moreover, absence of well-defined molecular targets currently limits chemotherapy of TNBC in adjuvant or metastatic settings to cytotoxic agents (3), whereas newer targeted drugs have shown disappointing treatment responses (4). Hence, there is a real need to develop new therapeutic agents that can improve the survival and quality of life of patients with TNBC (5).

A compound that shows promising therapeutic action on TNBC is curcumin, a natural polyphenol with a wide range of anti-cancer properties that has the added advantage of minimal side effects $(4,6,7)$. In a study using MDA-MB-231 breast cancer cells, a cell model widely used to develop candidate drugs against TNBC, curcumin was demonstrated to effectively inhibit cell proliferation and induce apoptosis (6). However, despite the promising anti-tumor properties of curcumin in vitro, very low aqueous solubility and poor bioavailability is a major obstacle for its successful use in vivo (8). Furthermore, curcumin is highly unstable and is rapidly degraded with a short half-life following administration into living bodies. These undesirable in vivo properties render it impractical to deliver curcumin as a free compound to target tissue in pharmacological concentrations (9).

Biocompatible nanoparticle (NP) systems are being extensively investigated as vehicles to stabilize and deliver anti-tumor drugs in vivo. For example, our group previously formulated polyethylene glycol-polylactic acid (PEG-PLA) polymeric NPs loaded with resveratrol and confirmed improved metabolic and antitumor effects in vivo (10). Similar approaches have also been applied to improve the pharmacokinetics of curcumin. 
These include encapsulating the compound by conjugation to liposomes, polymeric NPs and micelles (11-13). However, whereas most NP drug-delivery systems developed to date rely on enhanced permeation and retention for passive tumor accumulation (14), newer delivery systems achieve greater treatment effects by actively targeting cancer cells. A highly promising therapeutic target for TNBC is the epidermal growth factor (EGF) receptor. EGF receptors play a crucial role in tumor growth, invasion, and metastasis (15), and are overexpressed in over half of TNBCs (16). We and others have previously targeted EGF receptor-overexpressing cancer cells with its cognate ligand, EGF (17-19). Growth factor peptides are the most suited for selective tracing of functionally active high-affinity surface receptors because they have greater binding affinity and better tumor penetration compared to antibodies. To our knowledge, there is only one previous study that synthesized an EGF receptor-targeted NP for curcumin delivery. Yan et al, recently prepared a poly (D, L-lactic acid-coglycolic acid)-block-PEG copolymer conjugated with a synthetic EGF peptide called GE11 for co-delivery of docetaxel and a curcumin prodrug (20). However, although significant antitumor effects were observed in prostate cancer bearing mice, this was mainly attributed to the effect of docetaxel rather than to the curcumin prodrug (20). Hence, there is a need to explore the efficacy of EGF receptor-targeted curcumin delivery NP systems for the treatment of TNBC.

In this study, we thus developed DSPE-PEG micelle NPs encapsulating curcumin for improved in vivo bioavailability. Functional groups of the NP were conjugated with EGF peptide for EGF receptor-specific targeting. We examined the anticancer effects of curcumin delivery using these NPs on MDA-MB-468 TNBC cells in vitro and MDA-MB-468 tumors in living mice.

\section{Materials and methods}

Cell lines and reagents. MDA-MB-468 human breast cancer cells from the American Type Culture Collection (ATCC; Manassas, VA, USA) were cultured in a humidified incubator at $37^{\circ} \mathrm{C}$ and $5 \% \mathrm{CO}_{2}$ in RPMI-1640 media. Media was supplemented with 10\% FBS, 2 mM L-glutamine, $100 \mathrm{U} / \mathrm{ml}$ penicillin, and $100 \mathrm{mg} / \mathrm{ml}$ streptomycin.

N-hydroxysuccinimide-PEG ${ }_{10000}$-DSPE (DSPE-PEG-NHS) was purchased from NANOCS Inc. (New York, NY, USA). Curcumin and tetrahydrofurane were from Sigma-Aldrich (Merck KGaA, Darmstadt, Germany). Human EGF was purchased from GenScript (Piscataway, NJ, USA), and SnakeSkin dialysis tubing was from Thermo Fisher Scientific, Inc. (Waltham, MA, USA).

Preparation of EGF-conjugated DSPE-PEG. The lysine residues of EGF peptides were conjugated to the NHS group of DSPE-PEG-NHS. Briefly, $10 \mathrm{mg} / \mathrm{ml}$ of EGF was mixed with $10 \mathrm{mg}$ of NHS-functionalized DSPE-PEG in 0.1 M HEPES buffer ( $\mathrm{pH} 7.8)$ at a molar ratio of 1:2. The mixture was reacted overnight with gentle shaking at room temperature (RT) and then transferred into a dialysis membrane with a molecular weight cut-off of $10 \mathrm{kDa}$. Unbound EGF was removed by $24 \mathrm{~h}$ dialysis with stirring and change of deionized water every 2 h. EGF-DSPE-PEG was finally lyophilized on a FDU-1200 freeze dryer (EYELA; Tokyo Rikakikai Co., Ltd., Tokyo, Japan) and stored at $-70^{\circ} \mathrm{C}$ until use.

Formulation of curcumin-loaded phospholipid NPs (Cur-NPs). EGF-conjugated and unconjugated DSPE-PEG phospholipid micelles were loaded with curcumin to formulate EGF-Cur-NP and Cur-NP, respectively, using the thin-film hydration method (21). Briefly, $2 \mathrm{mg}$ of curcumin and $20 \mathrm{mg}$ of EGF-conjugated or unconjugated DSPE-PEG were dissolved in $1 \mathrm{ml}$ tetrahydrofurane at RT and mixed in a round-bottom flask. The organic phase-solution mixture was evaporated under vacuum at $40^{\circ} \mathrm{C}$ for $30 \mathrm{~min}$. The film was flushed with nitrogen gas for $50 \mathrm{~min}$, and the organic solvent was completely removed overnight at RT in a fume-hood. The thin film was hydrated with $20 \mathrm{ml}$ of $0.5 \mathrm{mM}$ HEPES buffer ( $\mathrm{pH} 7.5$ ) with vortexing, and sonicated for $3 \mathrm{~min}$ in a $50^{\circ} \mathrm{C}$ water bath to form micelles. The NPs were purified by passage through a $0.22 \mu \mathrm{m}$ syringe filter to remove unloaded curcumin and transferred to a fresh $50 \mathrm{ml}$ tube. The resultant nano-suspension was cooled to $-70^{\circ} \mathrm{C}$, lyophilized by a freeze dryer, and stored at $-70^{\circ} \mathrm{C}$ until use. Empty NP was prepared by the same procedure using unconjugated DSPE-PEG without curcumin loading.

Physicochemical characterization of NPs. The hydrodynamic diameter of NPs was determined with a DLS-7000 dynamic light scattering spectrophotometer (Brookhaven Instruments, Corporation, Holtsville, NY, USA) at $25^{\circ} \mathrm{C}$ using a scattering angle of $90^{\circ}$. The zeta potential of NPs in filtered phosphate-buffered saline (PBS; pH 7.4) was measured by a ZetaPlus analyzer (Brookhaven Instruments, Corporation). Measurements were performed twice per sample. The morphological shape of NPs was assessed by a JEM ARM 200F transmission electron microscope (JEOL, Peabody, MA, USA). Briefly, a drop of NPs was placed on a Formvar-coated copper grid, negatively stained with $1 \%$ uranyl acetated solution, and air-dried at RT. Magnifications of 50,000- to 100,000-fold were used.

Confirmation of EGF conjugation by EGF receptor activation. EGF conjugation of NPs was confirmed by its ability to activate EGF receptors on overexpressing MDA-MB-468 cancer cells by 5 min stimulation with a concentration of $5 \mathrm{nM}$. Free EGF and Cur-NP were used as positive and negative controls, respectively. Cells were lysed, and extracted protein was separated on a $10 \%$ sodium dodecyl sulfate polyacrylamide gel electrophoresis and transferred to a polyvinylidene difluoride membrane. After incubation with a rabbit monoclonal antibody against total EGFR or a mouse monoclonal antibody against phospho-EGFR (Tyr1068; Cell Signaling Technology, Inc., Danvers, MA, USA) at $4^{\circ} \mathrm{C}$ overnight, the membrane was incubated with a secondary antibody at RT for $1 \mathrm{~h}$. Immuno-reactive protein was detected by chemiluminescence, and band intensities were quantified using a GS-800 densitometer and Quantity One software (Bio-Rad Laboratories, Inc., Hercules, CA, USA).

Radioiodine labeling of NPs and cell binding assays. Cur-NP and EGF-Cur-NP were radiolabeled with ${ }^{125} \mathrm{I}$ using the Iodo-gen technique. Briefly, Pierce pre-coated IODO-GEN tubes (Thermo Fisher Scientific, Inc.) were washed with PBS, 
A

\begin{tabular}{ccc} 
& Cur-NP & EGF-Cur-NP \\
\hline Particle size $(\mathrm{nm})$ & $248.9 \pm 2.8$ & $229.3 \pm 6.0$ \\
P.I. & $0.17 \pm 0.04$ & $0.20 \pm 0.02$
\end{tabular}

Zeta potential $(\mathrm{mV}) \quad-3.60 \pm 2.3 \quad-1.73 \pm 2.2$

B
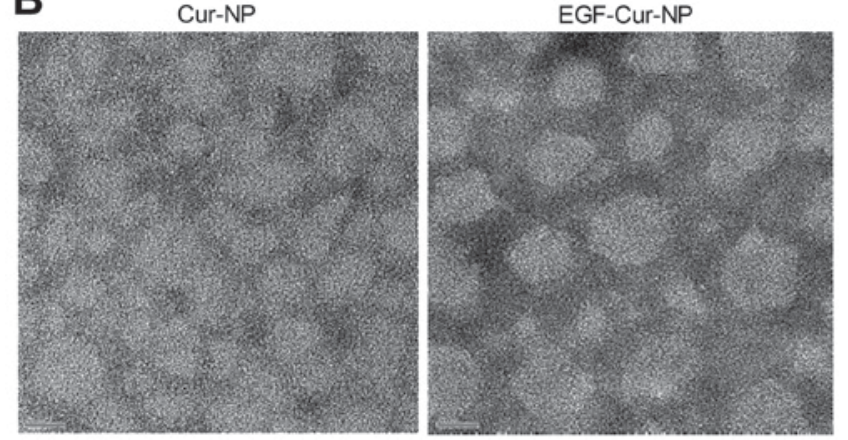

Figure 1. Characteristics and morphologies of curcumin-loaded DSPE-PEG NP formulations. (A) Particle size, zeta potential, and polydispersity index of Cur-NP and EGF-Cur-NP formulations. (B) TEM images of Cur-NP and EGF-Cur-NP. Scale bar=20 nm; TEM magnification, $x 100,000$. EGF, epidermal growth factor; Cur, curcumin; NP, nanoparticle; DSPE, 1,2-Distearoyl-sn-Glycero-3-Phosphoethanolamine; PEG, polyethylene glycol.

after which $100 \mu \mathrm{l}$ PBS, $17.5 \mathrm{MBq}(5 \mu \mathrm{l})$ of $\mathrm{Na}^{125} \mathrm{I}$, and $50 \mu \mathrm{l}$ of NPs (250 $\mu \mathrm{M}$ stock) were added. After 30 min reaction, the mixture was loaded on a PBS pre-soaked PD-10 desalting column. PBS elution was performed to separate ${ }^{125} \mathrm{I}$-labeled NP from free ${ }^{125} \mathrm{I}$ by collecting $0.5 \mathrm{ml}$ fractions that were measured for radioactivity. The first radioactivity peak fraction was used for cellular uptake experiments.

For binding experiments, cancer cells were incubated for $1 \mathrm{~h}$ with $110 \sim 150 \mathrm{kBq}$ of ${ }^{125}$ I-Cur-NP or ${ }^{125}$ I-EGF-Cur-NP in Dulbecco-PBS with $1 \%$ bovine serum albumin (BSA) at $37^{\circ} \mathrm{C}$ and $5 \% \mathrm{CO}_{2}$. EGF receptor-specific binding was determined by adding an excess amount of cold EGF $(10 \mu \mathrm{M})$. Cells were rapidly washed twice and measured for cell-associated radioactivity on a $\gamma$-counter (Wallac; PerkinElmer, MA, USA). Uptake levels were expressed as radio-counts relative to that of controls.

Fluorescent microscopic evaluation of curcumin internalization. MDA-MB-468 cells grown on an 8-well chamber slide (Lab-Tek II; Nalge Nunc International, Rochester, NY, USA) to $80 \%$ confluence were treated for $2 \mathrm{~h}$ with $10 \mu \mathrm{M}$ of free curcumin, Cur-NP, or EGF-Cur-NP. Cells were then washed twice with PBS and CC/Mount aqueous solution mounted (Sigma-Aldrich; Merck KGaA). Curcumin, which fluoresces at approximately $405 \mathrm{~nm}$, was visualized within cells by a Zeiss confocal laser scanning microscope using appropriate filters.

In vitro cytotoxicity and colony formation assay. Cytotoxic effects were evaluated by sulforhodamine B (SRB) assays. MDA-MB-468 cells were seeded on a 96-well plate at a density of $4 \times 10^{3}$ cells per well and treated $24 \mathrm{~h}$ later with graded doses of free curcumin, Cur-NP, or EGF-Cur-NP for 72 h. Cells were then fixed with $10 \%(\mathrm{w} / \mathrm{v})$ trichloroacetic acid and stained with SRB for $30 \mathrm{~min}$. Excess dye was removed by repeated washing with $1 \%(\mathrm{v} / \mathrm{v})$ acetic acid, and protein-bound dye was dissolved in $10 \mathrm{mM}$ Tris solution for optical density determination at $510 \mathrm{~nm}$ on a micro-plate reader.

Colony formation assays were performed on MDA-MB-468 cells seeded on a 6-well plate at a density of 400 cells per well. Cells were treated $24 \mathrm{~h}$ later with $5 \mu \mathrm{M}$ of free Cur, Cur-NP, or EGF-Cur-NP for $72 \mathrm{~h}$. Media was changed every 3 days for two weeks until colony formation. Cells were finally washed twice with cold PBS and stained with $2.3 \%$ (w/v) crystal violet solution for $30 \mathrm{~min}$. After staining, wells were rinsed with tap water, dried in room air at RT, and the number of colonies containing $>50$ cells was counted.

Tumor-bearing mouse model and NP treatment. All animal experiments were performed in accordance with the National Institutes of Health Guide for the Care and Use of Laboratory Animals and approved by the Samsung Biomedical Research Institute ethics committee. Tumor models were prepared in BALB/c nude mice by subcutaneous injection of $5 \times 10^{6}$ MDA-MB- 468 breast cancer cells into the right shoulder. When tumor size averaged $50 \mathrm{~mm}^{3}$, mice were randomly allocated into empty NP, Cur-NP (10 mg/kg), and EGF-Cur-NP $(10 \mathrm{mg} / \mathrm{kg}$ ) treatment groups (all in $0.3 \mathrm{ml}$ saline, $\mathrm{n}=4$ per group). NPs were intraperitoneally injected three times per week for a total of 8 injections. Health status of the mice was monitored by observation of behavior and weighing of body weight. Tumors were measured each treatment day with a caliper for maximal (length) and minimal diameter (width). Tumor volume in $\mathrm{mm}^{3}$ was calculated as length $\mathrm{x} \mathrm{width}^{2} \mathrm{x} 1 / 2$.

Invivo imaging of tumor ${ }^{18} \mathrm{~F}-\mathrm{FDG}$ uptake. A pilotmicro-PET/CT imaging test was performed in 2 separate tumor-bearing mice fasted for $4 \mathrm{~h}$ immediately prior to and $24 \mathrm{~h}$ after the first dose of either vehicle (saline) or EGF-Cur-NP ( $n=1$, each). At $1 \mathrm{~h}$ after tail vein injection with $7.4 \mathrm{MBq}$ of ${ }^{18} \mathrm{~F}-\mathrm{FDG}$, animals were isoflurane anesthetized, and PET/CT images were acquired on an Inveon scanner (Siemens Medical Solutions, Erlangen, Germany). On non-attenuation-corrected coronal PET images, ovoid regions-of-interest (ROIs) were placed to include all tumor mass while excluding adjacent tissue. A 20\% threshold was then used to automatically delineate the tumor margin. A second ROI was drawn on the contralateral shoulder as background activity. Tumor-to-background $(\mathrm{Tm} / \mathrm{Bkg})$ ratios of uptake were obtained by dividing mean standard uptake value of the tumor by that of background.

Data analysis. All data are presented as mean \pm standard deviation. Student's t-tests were used to compare 2 groups, and two-way analysis of variance with Fisher's least significant difference post hoc analysis was used to compare 3 or more groups. $\mathrm{P}<0.05$ was considered to indicate a statistically significant difference.

\section{Results}

Characteristics of curcumin-loaded NPs. Phospholipid NPs successfully formed micellar structures by thin-film 
A

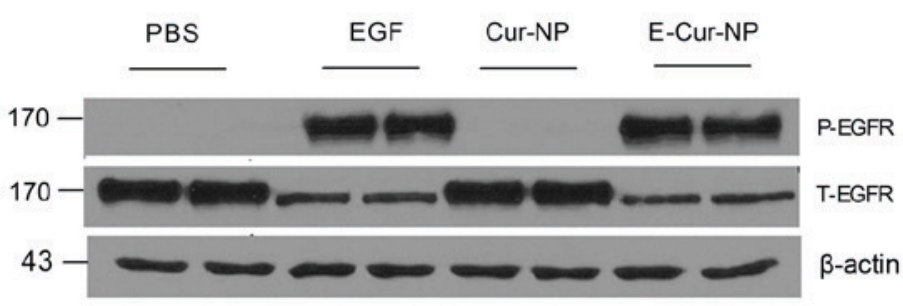

B

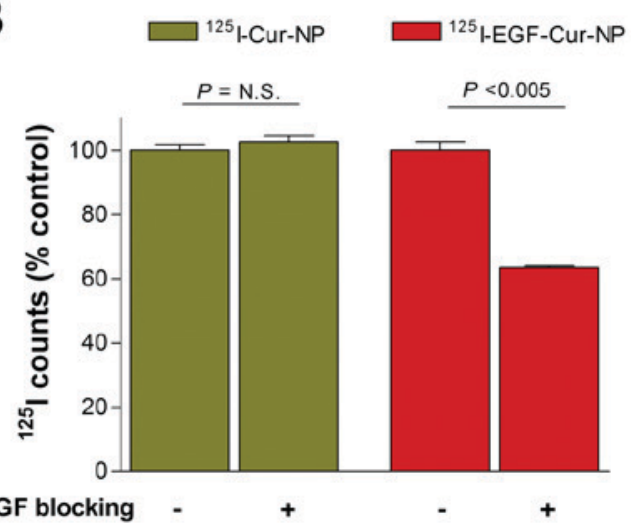

Figure 2. EGF receptor targeting of EGF-Cur-NP. (A) Immunoblotting for phosphorylated EGF receptor (shown at $170 \mathrm{kDa}$ ) shows that EGF-Cur-NP efficiently activates these receptors. (B) ${ }^{125}$ I-Cur-NPs and ${ }^{125}$ I-EGF-Cur-NPs binding to MDA-MB-468 TNBC cells. EGF receptor-specific binding was assessed by adding excess cold EGF $(10 \mu \mathrm{M})$. Bars are mean $\pm \mathrm{SD}$ of $\%$ binding relative to cells without blocking obtained from duplicate samples per group. (P=N.S., non-significance, $\mathrm{P}<0.005$ ). EGF, epidermal growth factor; Cur, curcumin; NP, nanoparticle.

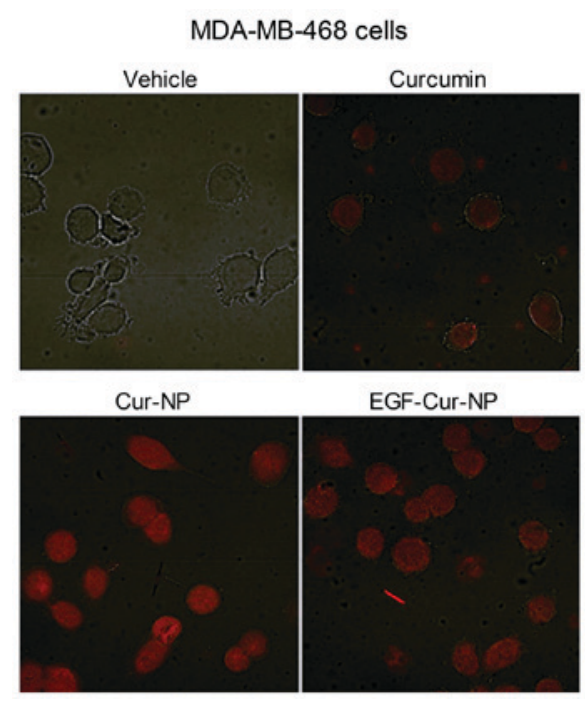

Figure 3. Fluorescence microscopic imaging of free curcumin, Cur-NP, and EGF-Cur-NP taken up by EGFR over-expressing MDA-MB-468 breast cancer cells. Magnification, x400. EGF, epidermal growth factor; Cur, curcumin; NP, nanoparticle.

hydration with a curcumin loading efficiency of $63.3 \%$. Whereas free curcumin was extremely hydrophobic, Cur-NP and EGF-Cur-NP showed good solubility in PBS. The physicochemical characteristics of Cur-NP and EGF-Cur-NP are summarized in Fig. 1A. Mean diameter measured by dynamic light scattering was $248.9 \pm 2.8 \mathrm{~nm}$ for Cur-NP and $229.3 \pm 6.0 \mathrm{~nm}$ for EGF-Cur-NP. Zeta potential was close to neutral and similar for Cur-NP and EGF-Cur-NP (Fig. 1A). Particle morphology assessed by transmission electron microscopy displayed evenly formulated Cur-NP and EGF-Cur-NP without clustering (Fig. 1B). These findings demonstrate that EGF conjugation does not significantly influence Cur-NP size, zeta potential, or morphology.

EGF receptor-specific targeting of EGF-Cur-NP. Western blotting of overexpressing MDA-MB-468 cells showed that 5 min incubations with $5 \mathrm{nM}$ of free EGF or EGF-Cur-NP were similarly potent stimulators for EGF receptor activation (phosphorylation), whereas incubation with Cur-NP was not (Fig. 2A).

Competitive binding assays further confirmed EGF receptor specificity of ${ }^{125}$ I-EGF-Cur-NP binding. Whereas ${ }^{125}$ I-Cur-NP binding to MDA-MB-468 cells was not influenced by excess EGF, ${ }^{125}$ I-EGF-Cur-NP binding was significantly reduced to $63.5 \pm 0.7 \%$ of that of the controls in the presence of $10 \mu \mathrm{M}$ of unlabeled EGF (Fig. 2B).

Fluorescent microscopy of NP-mediated curcumin delivery into cancer cells. The efficiency of cellular delivery of curcumin by NPs was compared to free curcumin by visualizing fluorescent signals from the drug under confocal microscopy. The results showed uniform intracellular delivery by curcumin-loaded NP and EGF-NP that was more efficient as than free curcumin for MDA-MB-468 cells (Fig. 3).

In vitro effect of Cur-NPs and EGF-Cur-NPs on cancer cell survival. In vitro cytotoxicity assays on MDA-MB-468 cells showed that only the highest dose tested $(10 \mu \mathrm{M})$ of free curcumin and Cur-NP significantly reduced cell viability to $41.5 \pm 2.8$ and $63.1 \pm 8.3 \%$ of baseline levels, respectively (Fig. 4A). In comparison, EGF-Cur-NPs exerted a substantially greater dose-dependent antitumor effect that began at a dose of $312 \mathrm{nM}$ and further suppressed cell survival to $12.2 \pm 3.0 \%$ of baseline level with a dose of $10 \mu \mathrm{M}$ (Fig. 4A). The calculated half inhibitory concentration (IC50) for EGF-Cur-NP was $620 \mathrm{nM}$.

The ability to suppress MDA-MB-468 cancer cell colony formation was also greatest for EGF-Cur-NPF compared to Cur-NP or free curcumin. Hence, treatment with $5 \mu \mathrm{M}$ of free curcumin and Cur-NP for $72 \mathrm{~h}$ reduced colony number over 2 weeks to $36.9 \pm 7.7$ and $13.5 \pm 1.5 \%$ of controls, respectively (Fig. 4B). Treatment with $5 \mu \mathrm{M}$ of EGF-Cur-NPs completely abrogated the colony forming activity of the cells (Fig. 4B).

Effects of treatment with NPs on in vivo tumor growth. Piloting of the metabolic response of MDA-MB-468 tumors to EGF-Cur-NP treatment using micro-PET/CT images showed a $48.0 \%$ reduction of tumor-to-background ratio of ${ }^{18} \mathrm{~F}-\mathrm{FDG}$ uptake compared to baseline level after a single dose of 


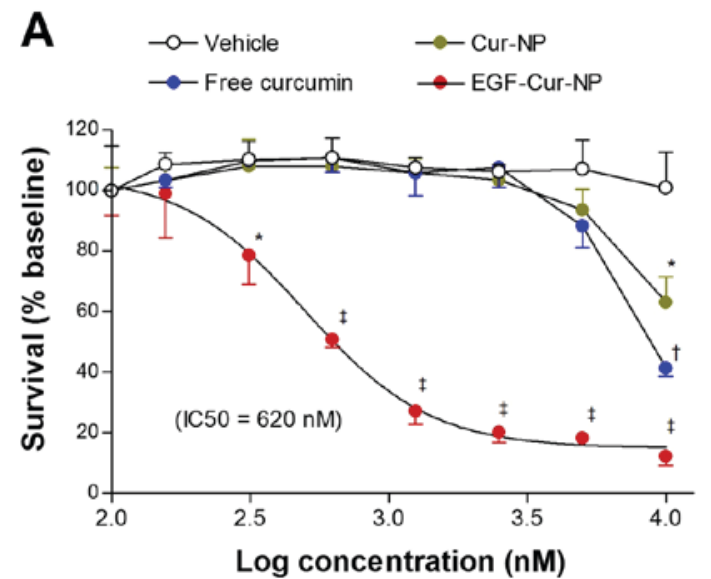

B

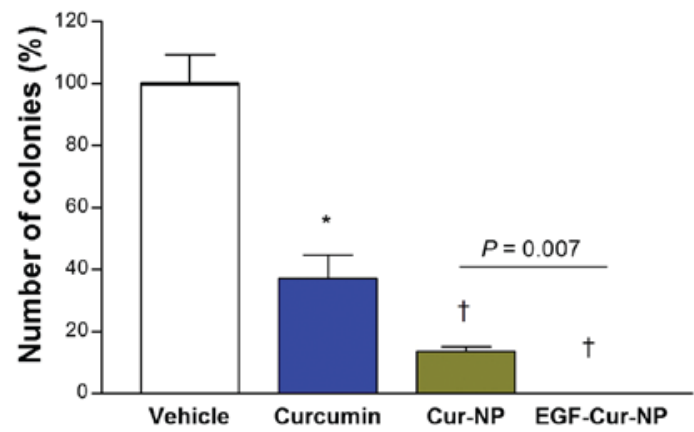

Figure 4. Comparison of cytotoxic effects on MDA-MB-468 cancer cells. (A) SRB assay-based viable cell content following $72 \mathrm{~h}$ treatment with vehicle (0.5\% DMSO) or graded concentrations of free curcumin, Cur-NP, or EGF-Cur-NP. (B) Number of cancer cell colonies ( $>50$ cells) assessed by crystal violet staining. Following $72 \mathrm{~h}$ treatment with vehicle or $5 \mu \mathrm{M}$ free curcumin, Cur-NP, or EGF-Cur-NP, media was freshly changed, and cells were further cultured for 2 weeks. Data are mean $\pm \mathrm{SD}$ of 3 (A) or 2 (B) samples per group expressed as \% relative to vehicle-treated controls. (" $\mathrm{P}<0.05$, ${ }^{\dagger} \mathrm{P}<0.005$, ${ }^{\star} \mathrm{P}<0.001$, compared to vehicle-treated controls). EGF, epidermal growth factor; Cur, curcumin; NP, nanoparticle; SRB, sulforhodamine B.
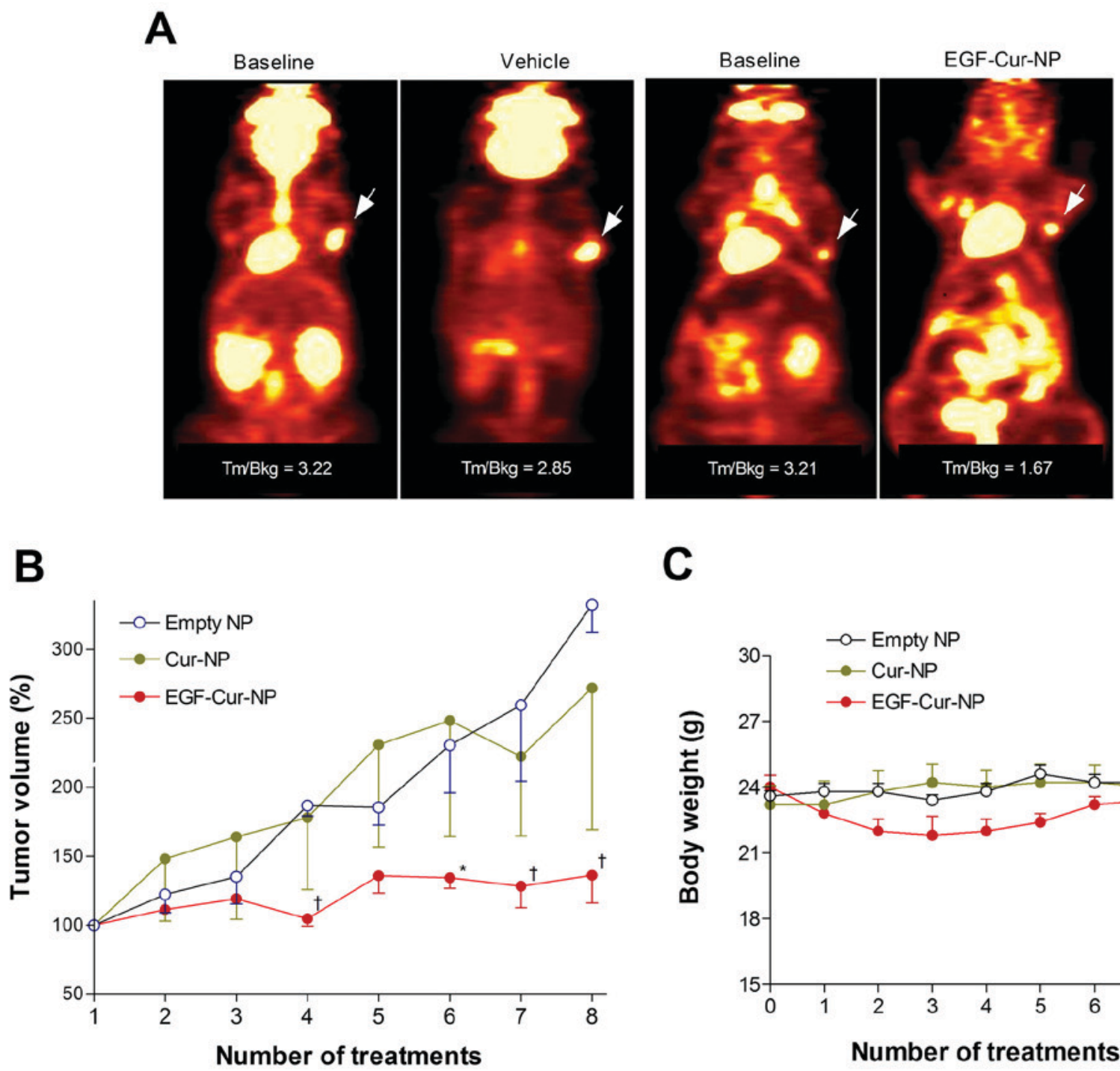

C

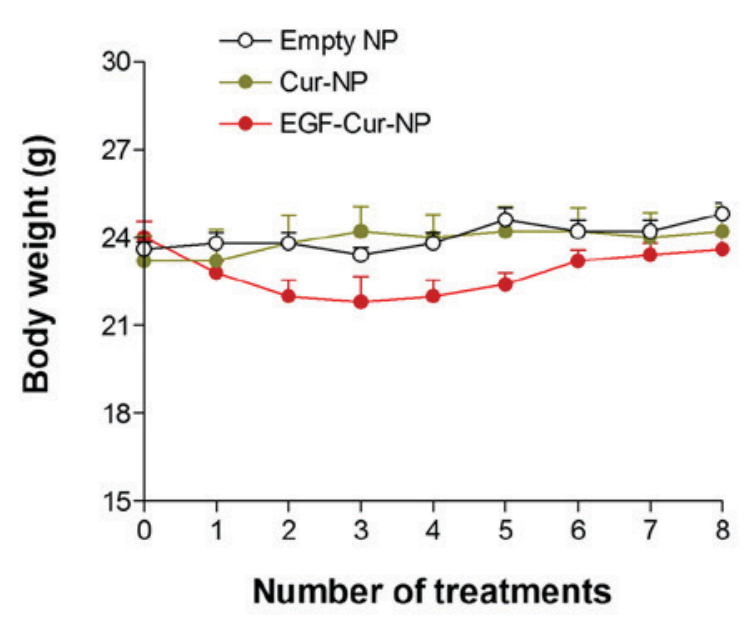

Figure 5. Metabolic and anti-tumor effects of NP formulations. (A) Micro-PET/CT images of mice showing ${ }^{18} \mathrm{~F}-\mathrm{FDG}$ uptake of MDA-MB-468 tumor (arrows) at baseline and $24 \mathrm{~h}$ after the single intravenous injection of vehicle or $10 \mathrm{mg} / \mathrm{kg}$ EGF-Cur-NP. Tumor-to-background ratio (Tm/Bkg) of ${ }^{18} \mathrm{~F}-\mathrm{FDG}$ uptake is shown for each study. (B) Tumor volume in MDA-MB-468 tumor-bearing mice during repeated treatment with empty NP, Cur-NP, or EGF-Cur-NP. Data are mean $\pm \mathrm{SD}$ of $\%$ volume obtained from 4 animals per group. " $\mathrm{P}<0.05$; ${ }^{\dagger} \mathrm{P}<0.005$ compared to empty NP injected controls. (C) Body weights of mice during repeated treatment. EGF, epidermal growth factor; Cur, curcumin; NP, nanoparticle. 
EGF-Cur-NP, while saline injection caused only a mild $11.5 \%$ reduction of ${ }^{18} \mathrm{~F}$-FDG uptake (Fig. 5A).

Finally, the therapeutic effects of repeated NP administration on MDA-MB-468 tumor-bearing mice were evaluated. Negative control animals administered empty NPs showed a steady growth of tumors to $400 \pm 1.5 \%$ of baseline volume over 3 weeks ( 8 repeat injections). Treatment with Cur-NP did not significantly reduce tumor growth compared to controls (Fig. 5B). In comparison, treatment with EGF-Cur-NP significantly retarded tumor growth compared to controls from the 4 to 8 th injections (Fig. 5B). After 8 injections, EGF-Cur-NP caused a $59.1 \%$ retardation of tumor growth compared to empty NP. The results showed no body-weight change in mice treated with empty NP or Cur-NP. The body-weight of mice treated with EGF-Cur-NP showed a transient mild (9.2\%) reduction at mid treatment $(\mathrm{P}<0.01$; two-way repeat measures ANOVA), but recovered to the level of other groups by the end of treatment (Fig. 5C).

\section{Discussion}

Despite promising anti-tumor effects in vitro, the clinical application of curcumin in patients with cancer including TNBC is severely hampered by difficulty in achieving pharmacologic concentrations in target tissue due to poor aqueous solubility and rapid degradation in vivo (8).

In this study, we prepared phospholipid NPs to load curcumin for improved solubility and in vivo stability. Phospholipids are naturally occurring amphiphilic molecules that constitute major structural elements of biological membranes. Because they are composed of hydrophilic and hydrophobic parts, phospholipid molecules self-assemble to form micellar NP shells. These NPs can trap hydrophobic drugs for stable delivery in vivo, with avoidance of plasma protein adsorption and prolonged circulation time (22). In our study, DSPE-PEG-NHS phospholipid NPs self-assembled into micelles and could load curcumin in a straightforward and efficient manner through thin-film hydration. NPs administered in excessive amounts can potentially exert adverse effects on cellular physiology by reactive oxygen species generation, cytokine secretion or inflammatory responses (23). The in vivo toxicity of NPs is highly dependent not only on dosage, but also on their chemical composition and physicochemical properties. The NPs used in this study were biodegradable phospholipid micelles that have more favorable safety profiles compared to less biocompatible NPs (24).

In NP formulation, the major challenges to curcumin are low aqueous solubility and poor in vivo stability (8). We also found that curcumin was insoluble in PBS and needed to be dissolved in DMSO or ethanol. In contrast, our curcumin-encapsulated DSPE-PEG formulation was readily dissolved in PBS, which indicates improved bioavailability under systemic administration. Although stability tests were not performed in the present study, a previous study showed that curcumin encapsulated in DSPE-PEG phospholipid was highly stable in PBS for up to $8 \mathrm{~h}$, whereas more than half of free curcumin was degraded within $10 \mathrm{~min}(25)$.

Although most nanometer-sized NPs for drug delivery are designed to reach tumor cells via leaky tumor vasculature (26), ligand conjugation allows specific targeting for increased drug accumulation into tumor tissue. We therefore conjugated NHS-functionalized PEG molecules with amine residues of EGF peptide to obtain curcumin-loaded NPs with EGF receptor-specific targeting capacity. Physicochemical characteristics of curcumin-loaded NPs were not significantly altered by EGF ligand conjugation, and specific binding to and activation of EGF receptors expressed on MDA-MB-468 TNBC cells were confirmed by Western blots and competitive binding assays, respectively.

Intracellular uptake and localization of curcumin after treatment with the free drug or with delivery vehicles can be quantitatively assessed by laser confocal microscopy using certain absorption and fluorescence wavelengths (27). In our study, homogenous distribution of fluorescence demonstrated that curcumin was located uniformly within cancer cells. The intensity of fluorescent signals from curcumin was significantly higher in MDA-MB-468 TNBC cells treated with curcumin-loaded NPs compared to those treated with free curcumin. Based on the previously verified linear dependency of fluorescence intensity on intracellular concentration level (27), this finding indicates greater cellular uptake and/or retention when curcumin is delivered via our NPs.

Despite numerous studies on the cytotoxic effects of curcumin on malignant cells, there is a remarkable paucity of reports on its effects on normal cells. Although curcumin has been shown capable of exerting cytotoxicity to normal cells as well as cancer cells (28), it is suggested that tumor cells have significantly greater sensitivity (27). In the case of curcumin NPs, even high doses are considered extremely safe in vivo, making it suitable as an anticancer agent with minimal toxicity to normal tissues (29). The NPs used in this study may further reduce adverse effects on normal tissues because PEG conjugation decreases uptake by cells of the reticulo-endothelial system while EGF conjugation allows selective targeting of cancer cells over normal cells.

Curcumin is reported to negatively regulate various growth factors, protein kinases, transcription factors, cell receptors and oncogenic proteins. Meanwhile the compound can induce cell cycle arrest or apoptotic death of malignant cells $(30,31)$. In our in vitro cytotoxicity experiments, EGF-Cur-NPs potently suppressed MDA-MB-468 cancer cell survival, whereas the effects of free curcumin and Cur-NP were weak. Taurin et al previously treated MDA-MB-468 cells with polystyrene-co-maleic acid micelles encapsulating a curcumin analogue (RL71) and observed similar cytotoxicity compared to free drug (IC50, 1.05 vs. $0.98 \mu \mathrm{M}$ ) (32). In our results, EGF-Cur-NP demonstrated a stronger cytotoxic effect (IC50, $0.62 \mu \mathrm{M}$ ). Colony forming assays similarly demonstrated a significantly greater anti-tumor effect by EGF-Cur-NP, which completely abrogated the ability of MDA-MB-468 cells to form colonies. The number of colonies was also significantly reduced by Cur-NP and free curcumin, but to a lesser extent.

We finally compared the anti-tumor effects of our NPs in MDA-MB-468 tumor-bearing mice. We chose intraperitoneal injection for our NPs because this allows administration of larger volumes without burdening the cardiovascular system and has the advantage of longer circulation time with lower 
liver uptake compared to intravenous injection (33). Although our group previously showed that curcumin can shift cancer cell metabolism toward glycolytic flux (7), pilot experiments in the present study suggested reduced MDA-MB-468 tumor glucose metabolism by EGF-Cur-NP. Because the expected increase of tumor ${ }^{18} \mathrm{~F}$-FDG uptake was not observed, we did not attempt to measure the precise magnitude of the metabolic effect of EGF-Cur-NP treatment.

$\mathrm{Yu}$ et al previously tested the in vivo anti-tumor effects in mice repeatedly intravenously injected with $40 \mathrm{mg} / \mathrm{kg}$ of methyl ether PEG-poly lactide amphiphilic block copolymers loaded with curcumin and observed a $47.1 \%$ growth reduction of MCF-7 tumors (34). We used a much lower dose of $10 \mathrm{mg} / \mathrm{kg}$ for curcumin-loaded EGF-Cur-NPs and still observed a greater $59.1 \%$ reduction of MDA-MB-468 tumor growth compared to control animals.

In conclusion, DSPE-PEG phospholipid NPs conjugated with EGF can effectively target and activate EGF receptors expressed on TNBC cells. EGF-DSPE-PEG efficiently encapsulates curcumin to exert cytotoxic effects in vitro and antitumor effects in vivo in a manner superior to that of non-targeted Cur-NP.

\section{Acknowledgements}

Not applicable.

\section{Funding}

This work was supported by the Basic Science Research Program through the National Research Foundation of Korea (NRF) funded by the Ministry of Science, ICT, \& Future Planning (grant no. 2014R1A1A3050612).

\section{Availability of data and materials}

All data generated or analyzed during this study are included in this published article.

\section{Authors' contributions}

KHL and KHJ designed experiments and analyzed data; JHL, JWP and DHK performed experiments. SHM and YSC analyzed the data. KHJ and KHL wrote the manuscript. All authors read, provided feedback and approved the manuscript.

\section{Ethics approval and consent to participate}

All animal experiments were performed in accordance with the National Institutes of Health Guide for the Care and Use of Laboratory Animals and approved by the Samsung Biomedical Research Institute ethics committee.

\section{Consent for publication}

Not applicable.

\section{Competing interests}

The authors declare that they have no competing interests.

\section{References}

1. Anders CK and Carey LA: Biology, metastatic patterns, and treatment of patients with triple-negative breast cancer. Clin Breast Cancer 9 (Suppl 2): S73-S81, 2009.

2. Haffty BG, Yang Q, Reiss M, Kearney T, Higgins SA, Weidhaas J, Harris L, Hait W and Toppmeyer D: Locoregional relapse and distant metastasis in conservatively managed triple negative early-stage breast cancer. J Clin Oncol 24: 5652-5657, 2006.

3. Palma G, Frasci G, Chirico A, Esposito E, Siani C, Saturnino C, Arra C, Ciliberto G, Giordano A and D'Aiuto M: Triple negative breast cancer: Looking for the missing link between biology and treatments. Oncotarget 6: 26560-26574, 2015.

4. Shindikar A, Singh A, Nobre M and Kirolikar S: Curcumin and resveratrol as promising natural remedies with nanomedicine approach for the effective treatment of triple negative breast cancer. J Oncol 2016: 9750785, 2016.

5. Jamdade VS, Sethi N, Mundhe NA, Kumar P, Lahkar M and Sinha N: Therapeutic targets of triple-negative breast cancer: A review. Br J Pharmacol 172: 4228-4237, 2015

6. Sun XD, Liu XE and Huang DS: Curcumin induces apoptosis of triple-negative breast cancer cells by inhibition of EGFR expression. Mol Med Rep 6: 1267-1270, 2012.

7. Jung KH, Lee JH, Park JW, Moon SH, Cho YS and Lee KH: Effects of curcumin on cancer cell mitochondrial function and potential monitoring with ${ }^{18} \mathrm{~F}-\mathrm{FDG}$ uptake. Oncol Rep 35: 861-868, 2016.

8. Anand P, Kunnumakkara AB, Newman RA and Aggarwal BB: Bioavailability of curcumin: Problems and promises. Mol Pharm 4: 807-818, 2007.

9. Yallapu MM, Jaggi M and Chauhan SC: Curcumin nanoformulations: A future nanomedicine for cancer. Drug Discov Today 17: 71-80, 2012.

10. Jung KH, Lee JH, Park JW, Quach CHT, Moon SH, Cho YS and Lee KH: Resveratrol-loaded polymeric nanoparticles suppress glucose metabolism and tumor growth in vitro and in vivo. Int J Pharm 478: 251-257, 2015.

11. Naksuriya O, Okonogi S, Schiffelers RM and Hennink WE: Curcumin nanoformulations: A review of pharmaceutical properties and preclinical studies and clinical data related to cancer treatment. Biomaterials 35: 3365-3383, 2014.

12. Cheng J, Teply BA, Sherifi I, Sung J, Luther G, Gu FX, Levy-Nissenbaum E, Radovic-Moreno AF, Langer R and Farokhzad OC: Formulation of functionalized PLGA-PEG nanoparticles for in vivo targeted drug delivery. Biomaterials 28 : 869-876, 2007.

13. Chan JM, Zhang L, Yuet KP, Liao G, Rhee JW, Langer R and Farokhzad OC: PLGA-lecithin-PEG core-shell nanoparticles for controlled drug delivery. Biomaterials 30: 1627-1634, 2009.

14. Wong C, Stylianopoulos T, Cui J, Martin J, Chauhan VP, Jiang W, Popovic Z, Jain RK, Bawendi MG and Fukumura D: Multistage nanoparticle delivery system for deep penetration into tumor tissue. Proc Natl Acad Sci USA 108: 2426-2431, 2011.

15. Baselga $\mathrm{J}$ and Arteaga $\mathrm{CL}$ : Critical update and emerging trends in epidermal growth factor receptor targeting in cancer. J Clin Oncol 23: 2445-2459, 2005.

16. Masuda H, Zhang D, Bartholomeusz C, Doihara H, Hortobagyi GN and Ueno NT: Role of epidermal growth factor receptor in breast cancer. Breast Cancer Res Treat 136: 331-345, 2012.

17. Jung KH, Choe YS, Paik JY and Lee KH: 99mTc-Hydrazinonicotinamide epidermal growth factor-polyethylene glycol-quantum dot imaging allows quantification of breast cancer epidermal growth factor receptor expression and monitors receptor downregulation in response to cetuximab therapy. J Nucl Med 52: 1457-1464, 2011.

18. Jung KH, Park JW, Paik JY, Quach CH, Choe YS and Lee KH: EGF receptor targeted tumor imaging with biotin-PEG-EGF linked to (99m)Tc-HYNIC labeled avidin and streptavidin. Nucl Med Biol 39: 1122-1127, 2012.

19. Li J, Chen L, Liu N, Li S, Hao Y and Zhang X: EGF-coated nano-dendriplexes for tumor-targeted nucleic acid delivery in vivo. Drug Deliv 23: 1718-1725, 2016.

20. Yan J, Wang Y, Jia Y, Liu S, Tian C, Pan W, Liu X and Wang H: Co-delivery of docetaxel and curcumin prodrug via dual-targeted nanoparticles with synergistic antitumor activity against prostate cancer. Biomed Pharmacother 88: 374-383, 2017. 
21. Zhao BJ, Ke XY, Huang Y, Chen XM, Zhao X, Zhao BX, Lu WL, Lou JN, Zhang $X$ and Zhang Q: The antiangiogenic efficacy of NGR-modified PEG-DSPE micelles containing paclitaxel (NGR-M-PTX) for the treatment of glioma in rats. J Drug Target 19: 382-390, 2011.

22. Weingart J, Vabbilisetty P and Sun XL: Membrane mimetic surface functionalization of nanoparticles: Methods and applications. Adv Colloid Interface Sci 197-198: 68-84, 2013

23. Pandey RK and Prajapati VK: Molecular and immunological toxic effects of nanoparticles. Int J Biol Macromol 107: 1278-1293, 2018.

24. Gothwal A, Khan I and Gupta U: Polymeric micelles: Recent advancements in the delivery of anticancer drugs. Pharm Res 33: 18-39, 2016.

25. Gülçür E, Thaqi M, Khaja F, Kuzmis A and Önyüksel H: Curcumin in VIP-targeted sterically stabilized phospholipid nanomicelles: A novel therapeutic approach for breast cancer and breast cancer stem cells. Drug Deliv Transl Res 3, 2013.

26. Perrault SD, Walkey C, Jennings T, Fischer HC and Chan WC: Mediating tumor targeting efficiency of nanoparticles through design. Nano Lett 9: 1909-1915, 2009.

27. Kunwar A, Barik A, Mishra B, Rathinasamy K, Pandey R and Priyadarsini KI: Quantitative cellular uptake, localization and cytotoxicity of curcumin in normal and tumor cells. Biochim Biophys Acta 1780: 673-679, 2008.

28. Strojny B, Grodzik M, Sawosz E, Winnicka A, Kurantowicz N, Jaworski S, Kutwin M, Urbańska K, Hotowy A, Wierzbicki M and Chwalibog A: Diamond nanoparticles modify curcumin activity: In vitro studies on cancer and normal cells and in ovo studies on chicken embryo model. PLoS One 11: e0164637, 2016
29. Yallapu MM, Jaggi M and Chauhan SC: Curcumin nanomedicine: A road to cancer therapeutics. Curr Pharm Des 19: 1994-2010, 2013.

30. Deguchi A: Curcumin targets in inflammation and cancer. Endocr Metab Immune Disord Drug Targets 15: 88-96, 2015.

31. Shanmugam MK, Rane G, Kanchi MM, Arfuso F, Chinnathambi A, Zayed ME, Alharbi SA, Tan BK, Kumar AP and Sethi G: The multifaceted role of curcumin in cancer prevention and treatment. Molecules 20: 2728-2769, 2015.

32. Taurin S, Nehoff H, Diong J, Larsen L, Rosengren RJ and Greish K: Curcumin-derivative nanomicelles for the treatment of triple negative breast cancer. J Drug Target 21: 675-683, 2013

33. Jung C, Kaul MG, Bruns OT, Dučić T, Freund B, Heine M, Reimer R, Meents A, Salmen SC, Weller H, et al: Intraperitoneal injection improves the uptake of nanoparticle-labeled high-density lipoprotein to atherosclerotic plaques compared with intravenous injection: a multimodal imaging study in ApoE knockout mice. Circ Cardiovasc Imaging 7: 303-311, 2014.

34. Yu Y, Zhang X and Qiu L: The anti-tumor efficacy of curcumin when delivered by size/charge-changing multistage polymeric micelles based on amphiphilic poly $(\beta$-amino ester) derivates. Biomaterials 35: 3467-3479, 2014.

This work is licensed under a Creative Commons Attribution-NonCommercial-NoDerivatives 4.0 International (CC BY-NC-ND 4.0) License. 